\title{
Metabolic engineering strategies for optimizing acetate reduction, ethanol yield and osmotolerance in Saccharomyces cerevisiae
}

\author{
Ioannis Papapetridis ${ }^{1}$, Marlous van Dijk ${ }^{1,2}$, Antonius J. A. van Maris ${ }^{1,3}$ and Jack T. Pronk ${ }^{1 *}$
}

\begin{abstract}
Background: Glycerol, whose formation contributes to cellular redox balancing and osmoregulation in Saccharomyces cerevisiae, is an important by-product of yeast-based bioethanol production. Replacing the glycerol pathway by an engineered pathway for $\mathrm{NAD}^{+}$-dependent acetate reduction has been shown to improve ethanol yields and contribute to detoxification of acetate-containing media. However, the osmosensitivity of glycerol non-producing strains limits their applicability in high-osmolarity industrial processes. This study explores engineering strategies for minimizing glycerol production by acetate-reducing strains, while retaining osmotolerance.

Results: GPD2 encodes one of two S. cerevisiae isoenzymes of $\mathrm{NAD}^{+}$-dependent glycerol-3-phosphate dehydrogenase (G3PDH). Its deletion in an acetate-reducing strain yielded a fourfold lower glycerol production in anaerobic, low-osmolarity cultures but hardly affected glycerol production at high osmolarity. Replacement of both native G3PDHs by an archaeal NADP ${ }^{+}$-preferring enzyme, combined with deletion of ALD6, yielded an acetate-reducing strain the phenotype of which resembled that of a glycerol-negative gpd $1 \Delta$ gpd2 $\Delta$ strain in low-osmolarity cultures. This strain grew anaerobically at high osmolarity ( $1 \mathrm{~mol} \mathrm{~L}^{-1}$ glucose), while consuming acetate and producing virtually no extracellular glycerol. Its ethanol yield in high-osmolarity cultures was 13\% higher than that of an acetatereducing strain expressing the native glycerol pathway.

Conclusions: Deletion of GPD2 provides an attractive strategy for improving product yields of acetate-reducing $S$. cerevisiae strains in low, but not in high-osmolarity media. Replacement of the native yeast G3PDHs by a heterologous $\mathrm{NADP}^{+}$-preferring enzyme, combined with deletion of $A L D 6$, virtually eliminated glycerol production in high-osmolarity cultures while enabling efficient reduction of acetate to ethanol. After further optimization of growth kinetics, this strategy for uncoupling the roles of glycerol formation in redox homeostasis and osmotolerance can be applicable for improving performance of industrial strains in high-gravity acetate-containing processes.
\end{abstract}

Keywords: Yeast, NADH, NADPH, Redox engineering, Acetic acid, Osmotic stress, Bioethanol

\section{Background}

By functionally replacing fossil-fuel-derived compounds, microbial production of chemicals and transport fuels can contribute to a transition to a sustainable, low-carbon global economy [1]. The total industrial production of fuel ethanol, which reached ca. 100 billion litres in

\footnotetext{
*Correspondence: j.t.pronk@tudelft.nl

${ }^{1}$ Industrial Microbiology Section, Department of Biotechnology, Delft

University of Technology, Van der Maasweg 9, 2629 HZ Delft, The Netherlands

Full list of author information is available at the end of the article
}

2015, is predicted to increase further [2]. The yeast Saccharomyces cerevisiae is the established microbial cell factory for conversion of starch- and sucrose-derived hexose sugars to ethanol, as it combines a high ethanol yield and productivity with robustness under process conditions [3-5]. Efforts in yeast strain improvement and process optimization of corn-starch and cane-sugarbased bioethanol productions have further improved product yields and productivity [5]. Furthermore, intensive metabolic and evolutionary engineering studies have yielded yeast strains capable of efficiently fermenting the 
pentose sugars xylose and arabinose, thus paving the way for yeast-based 'second-generation' bioethanol production from lignocellulosic hydrolysates [6-8].

In industrial bioethanol production, the carbohydrate feedstock represents the single largest cost factor [9]. Maximizing ethanol yield on sugar is therefore a key requirement, especially in second-generation processes, ethanol yields and productivity of which are generally still lower than those of first-generation processes [6-8]. Adequate yeast performance in lignocellulosic hydrolysates also requires tolerance to inhibitors that are released during biomass pre-treatment and hydrolysis [10-12]. Suboptimal ethanol yields in industrial processes are caused by formation of biomass and low-molecular-weight metabolites, of which glycerol accounts for a loss of up to $4 \%$ of the carbohydrate substrate [13]. Under anaerobic conditions, wild-type $S$. cerevisiae strains require glycerol formation to re-oxidize NADH formed during biosynthesis or during production of metabolites whose formation results in net $\mathrm{NADH}$ formation $[14,15]$. As the major compatible solute in S. cerevisiae, glycerol also plays a key role in osmotolerance $[16,17]$.

In S. cerevisiae, glycerol formation is initiated by reduction of the glycolytic intermediate dihydroxyacetone phosphate to glycerol-3-phosphate, a reaction catalysed by two isoenzymes of $\mathrm{NAD}^{+}$-dependent glycerol-3P dehydrogenase (G3PDH), Gpd1 and Gpd2 [18]. Glycerol$3 \mathrm{P}$ is subsequently hydrolysed to glycerol and inorganic phosphate by glycerol-3P phosphatase, isoenzymes of which are encoded by GPP1 and GPP2. Reoxidation of $1 \mathrm{~mol}$ of $\mathrm{NADH}$ through glycerol production requires $0.5 \mathrm{~mol}$ glucose and $1 \mathrm{~mol}$ ATP [18]. Several metabolic engineering strategies have demonstrated that altering redox-cofactor specificity of reactions in biosynthesis [13] or in sugar dissimilation [19] can be used to decrease glycerol formation from sugars.

GPD1 and GPD2 are differentially regulated, as transcriptional upregulation of GPD1 mainly occurs in response to osmotic stress, while regulation of GPD2 is linked to redox homeostasis [20-22]. Complete elimination of glycerol production by inactivation of both GPD1 and GPD2 has been reported to abolish anaerobic growth and to greatly increase osmosensitivity [20, $21,23]$. Anaerobic growth of $g p d 1 \Delta$ gpd2 $\Delta$ cultures can be restored by addition of an external electron acceptor, such as acetoin, to growth media [23]. Recently, S. cerevisiae strains have been engineered to use acetic acid or $\mathrm{CO}_{2}$ as external electron acceptors [24-28]. Functional expression of a heterologous acetylating-acetaldehyde dehydrogenase (A-ALD), together with the activities of the native acetyl-CoA synthetases and alcohol dehydrogenases, enabled the NADH-dependent reduction of acetic acid to ethanol [25]. $\mathrm{CO}_{2}$ is abundantly present in all industrial ethanol fermentation processes, while acetic acid is an important, ubiquitous inhibitor of yeast performance in lignocellulosic hydrolysates $[10,11]$. Use of acetic acid as an external 'redox sink' is highly attractive for second-generation bioethanol processes, since its reduction to ethanol not only increases product yields, but simultaneously contributes to detoxification of the fermentation medium $[25,29,30]$.

Although metabolic engineering has enabled the replacement of glycerol production by the reduction of acetic acid to ethanol, the increased osmosensitivity of $g p d 1 \Delta \operatorname{gpd} 2 \Delta$ strains has not been fully resolved. Process intensification of bioethanol production via the introduction of high-gravity fermentation processes will make osmotolerance ever more important [31-36]. Previous research on improving osmotolerance of gpd1 $1 \Delta$ gpd $2 \Delta$ strains explored production of alternative compatible solutes, including the polyols mannitol and sorbitol [37], trehalose [38-40] and proline [41, 42]. These alternative compounds, however, did not confer the same osmotolerance as glycerol. Evolutionary engineering of an acetate-reducing $g p d 1 \Delta$ gpd $2 \Delta$ strain yielded a strain that could grow anaerobically at $1 \mathrm{~mol} \mathrm{~L}^{-1}$ glucose without loss of acetate-reduction potential or ethanol yield [43]. However, the underlying genetic changes were not fully resolved [43]. Tuning the expression of the native G3PDH genes can lead to decreased glycerol production, but the resulting strains still require the production of considerable amounts of this by-product to maintain the redox-cofactor balance [34, 35, 44]. To uncouple the roles of glycerol formation in NADH reoxidation and osmotolerance in S. cerevisiae, it would be of interest to alter the redox-cofactor specificity of G3PDH. Specifically, making this reaction NADPH-dependent might uncouple glycerol production from $\mathrm{NAD}^{+}$regeneration. In such a scenario, $\mathrm{NAD}^{+}$could then be exclusively regenerated via acetate or $\mathrm{CO}_{2}$ reduction. Simultaneously, NADPHdependent formation of intracellular glycerol would enable the synthesis of a compatible solute with minimal losses in ethanol yield. Recently, an $\mathrm{NADP}^{+}$-preferring G3PDH, encoded by the gpsA gene from the thermophilic archaeon Archaeoglobus fulgidus was described [45]. Based on its unusual cofactor preference and, due to its thermophilic origin, anticipated low in vivo activity in yeast, we hypothesized that $A$. fulgidus GpsA might be an interesting candidate to replace the $\mathrm{NAD}^{+}$-dependent Gpd1 and Gpd2 enzymes in S. cerevisiae.

The goal of this study is to explore new metabolic engineering strategies for construction of acetate-reducing, osmotolerant S. cerevisiae strains with a minimal, nonzero production of glycerol. To this end, acetate-reducing strains with different configurations of the native 
glycerol production pathway, as well as strains in which GPD1 and GPD2 were replaced by A. fulgidus gpsA, were constructed. To construct acetate-reducing strains, the ethanolamine utilization protein of $E$. coli, encoded by eutE [Genbank: WP_001075673.1], was overexpressed, as it was previously shown to support near-wild-type anaerobic growth rates in a gpd1 $1 \Delta$ gpd $2 \Delta$ strain background [29]. The impacts of these engineering strategies on acetate reduction and glycerol production were quantitatively analysed in anaerobic bioreactor batch cultures grown on low- and high-osmolarity media.

\section{Methods}

\section{Strain propagation and maintenance}

All $S$. cerevisiae strains used in this study belong to the CEN.PK lineage [46] (Table 1). S. cerevisiae cultures were propagated in synthetic medium [47] containing $20 \mathrm{~g} \mathrm{~L}^{-1}$ glucose. E. coli DH5a cultures for plasmid cloning were propagated in LB medium $\left(10 \mathrm{~g} \mathrm{~L}^{-1}\right.$ Bacto tryptone, $5 \mathrm{~g} \mathrm{~L}^{-1}$ Bacto yeast extract, $5 \mathrm{~g} \mathrm{~L}^{-1} \mathrm{NaCl}$ ) containing $100 \mathrm{mg} \mathrm{L}^{-1}$ ampicillin. All strains were stored at $-80{ }^{\circ} \mathrm{C}$, after addition of sterile glycerol $\left(30 \% \mathrm{v} \mathrm{v}^{-1}\right)$ to growing cultures.

\section{Construction of expression cassettes and plasmids}

Plasmids used in this study are listed in Table 2. Plasmids-expressing chimeric gRNAs were used for CRISPR/ Cas9-mediated genome editing [48]. Unique Cas9-recognition sequences in GPD1, GPD2, SGA1 and ALD6 were selected as described previously [29]. PCR for construction of expression cassettes and diagnostic PCR were performed using Phusion Hot Start II High Fidelity DNA Polymerase and Dreamtaq polymerase (Thermo Scientific, Waltham, MA), respectively, according to the manufacturer's guidelines. For the construction of pUDR240,
Table 2 Plasmids used in this study

\begin{tabular}{lll}
\hline Plasmid & Characteristics & Origin \\
\hline p426-TEF (empty) & $2 \mu$, URA3, TEF1p-CYC1t & {$[75]$} \\
pMEL10 & $2 \mu$, KIURA3, SNR52p-gRNA.CAN1.Y- & {$[48]$} \\
& SUP4t & \\
pMEL11 & $2 \mu$, amdS, SNR52p-gRNA.CAN1.Y-SUP4t & {$[48]$} \\
pROS10 & KIURA3-gRNA.CAN1-2mu-gRNA.ADE2 & {$[48]$} \\
pUDI076 & pRS406-TDH3p-eutE-CYC1t & {$[29]$} \\
pUDR103 & $2 \mu$, KIURA3, SNR52p-gRNA.SGA1.Y-SUP4t & This work \\
pUDR119 & $2 \mu$, amdS, SNR52p-gRNA.SGA1.Y-SUP4t & {$[76]$} \\
pUDR240 & KIURA3-gRNA.GPD1-2mu-gRNA.GPD2 & This work \\
pUDR264 & $2 \mu$, amdS, SNR52p-gRNA.ALD6.Y-SUP4t & This work \\
pMK-RQ-gpsA & Delivery vector, codon-optimized gpsA & GeneArt, \\
& ORF & Germany \\
\hline
\end{tabular}

the backbone of the plasmid was PCR amplified using the double-binding primer 5793 (Additional file 1) and pROS10 as template. The insert fragment, expressing the GPD1-targeting and GPD2-targeting gRNA cassettes, was amplified using primers $6965-6966$ and pROS10 as template. For the construction of pUDR103, the plasmid backbone of pMEL10 was PCR amplified using primers 5792-5980. The SGA1-targeting gRNA expression cassette was PCR amplified using primers $5979-7023$ and pMEL10 as template. For the construction of pUDR264, the plasmid backbone of pMEL11 was PCR amplified using primers 5792-5980. The ALD6-targeting gRNA expression cassette was PCR amplified using primers 5979-7610 and pMEL11 as a template. Plasmids were assembled with the Gibson Assembly Cloning kit (New England Biolabs, Ipswich, MA), after downscaling the supplier's protocol to $10 \mu \mathrm{L}$ reaction volumes. Plasmids pUDR240 and pUDR264 were cloned in E. coli DH5a

Table 1 Saccharomyces cerevisiae strains used in this study

\begin{tabular}{|c|c|c|}
\hline Strain name & Relevant genotype & Origin \\
\hline IMX585 & MAL2-8C SUC2 can1::cas9-natNT2 & [48] \\
\hline IMX581 & ura3-52 MAL2-8c SUC2 can 1:: cas9-natNT2 & [48] \\
\hline IMZ160 & ura3 leu2::LEU2 [pRS405] gpd1::IoxP gpd2::hphMX4 pUDE43 & [43] \\
\hline IME324 & ura3-52 MAL2-8c SUC2 can 1::cas9-natNT2 p426-TEF (empty) & This work \\
\hline IMX884 & ura3-52 MAL2-8c SUC2 can 1::cas9-natNT2 gpd2::eutE pROS10 (GPD2-targeting) & This work \\
\hline IMX992 & ura3-52 MAL2-8c SUC2 can 1::cas9-natNT2 sga1::eutE pUDR1 19 & This work \\
\hline IMX776 & ura3-52 MAL2-8c SUC2 can 1::cas9-natNT2 gpd1::.gpsA gpd2::eutE pUDR240 & This work \\
\hline IMX901 & 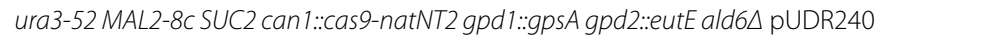 & This work \\
\hline IMX888 & MAL2-8c SUC2 can 1:: cas9-natNT2 gpd1 $\triangle$ gpd2::eutE & [29] \\
\hline IMX900 & MAL2-8c SUC2 can 1::cas9-natNT2 gpd1 $\triangle$ gpd2::eutE ald6 $\Delta$ & This work \\
\hline IMX1039 & ura3-52 MAL2-8c SUC2 can 1::cas9-natNT2 gpd1:::gpsA gpd2::eutE ald6 & This work \\
\hline $\operatorname{IMX} 1120$ & 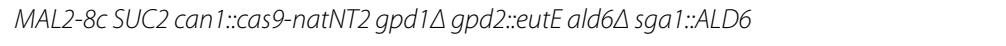 & This work \\
\hline $\operatorname{IMX} 1142$ & 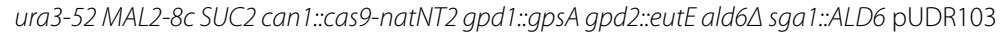 & This work \\
\hline
\end{tabular}


cells after transformation by electroporation and plasmid re-isolation with a miniprep kit (Sigma-Aldrich, St. Louis, $\mathrm{MO})$. Correct clones were verified by restriction digestion or by diagnostic PCR (DreamTaq polymerase, Additional file 1). For the single deletion of GPD2, a plasmid backbone was PCR amplified with the double-binding primer 5793 and pROS10 as template. The insert fragment, expressing two identical GPD2-targeting gRNA cassettes, was amplified using primer 6966 and pROS10 as templates. For the single deletion of GPD2, the two plasmid fragments were transformed directly into yeast cells and assembled in vivo.

An S. cerevisiae codon-optimized version of A. fulgidus gpsA [Genebank: AAB90367.1], based on the codon preference of highly expressed yeast glycolytic genes [49], was synthesized by GeneArt GmbH (Regensburg, Germany) [Genebank: KY554758]. An integration cassette for replacing the coding region of GPD1 by the codon-optimized gpsA sequence was PCR amplified with primers 7862-7863 and pMK-RQ-gpsA as template. Codon-optimized expression cassettes for the E. coli EutE acetylating-acetaldehyde dehydrogenase gene (TDH3peutE-CYC1t), aimed at integration in the GPD2 or SGA1 locus, were amplified with primers 7991-7992 or 72117025, respectively, using pUDI076 [29] as a template. A cassette expressing ALD6 from its native promoter and terminator sequences, aimed at integration in the $S G A 1$ locus, was amplified with primers 9809-9810, using genomic DNA of S. cerevisiae IMX581 as a template. Integration cassettes were flanked by 60 -bp sequences that enabled integration by homologous recombination after CRISPR/Cas9-mediated introduction of doublestrand breaks in selected S. cerevisiae genomic loci.

\section{Strain construction}

The lithium acetate/polyethylene glycol method [50] was used for yeast transformation. After transformation with plasmids pUDR103, pUDR240 and after single deletion of GPD2, transformants were selected on synthetic medium agar plates [47] containing $20 \mathrm{~g} \mathrm{~L}^{-1}$ glucose. After transformation with plasmids pUDR119 and pUDR264, selection and counter selection were performed as described [51]. Counter selection of plasmids carrying URA3 was performed on YP agar plates (10 $\mathrm{g} \mathrm{L}^{-1}$ Bacto yeast extract, $20 \mathrm{~g} \mathrm{~L}^{-1}$ Bacto peptone) supplemented with glucose ( $20 \mathrm{~g} \mathrm{~L}^{-1}$ final concentration) and 5-fluoroorotic acid (1 $\mathrm{g} \mathrm{L}^{-1}$ final concentration). Diagnostic colony PCR was used for genotypic analysis of selected colonies. Co-transformation of pUDR119 and the SGA1-flanked TDH3p-eutE-CYC1t cassette into strain IMX581 yielded strain IMX992, in which eutE was overexpressed in the presence of functional GPD1 and GPD2 genes. Co-transformation of the two fragments of the GPD2-targeting gRNA plasmid and the GPD2flanked TDH3p-eutE-CYC1t cassette to strain IMX581 yielded strain IMX884, in which GPD2 was deleted and eutE was overexpressed. Co-transformation of pUDR240, the GPD1-flanked gpsA coding sequence and the GPD2flanked TDH3p-eutE-CYC1t cassette to strain IMX581 yielded strain IMX776, in which gpsA was expressed from the native GPD1 promoter and terminator, GPD2 was deleted and eutE was overexpressed. Co-transformation of pUDR264 and the repair oligonucleotides 7608-7609, followed by pUDR264 counter selection, into strains IMX776 and IMX888 yielded strains IMX901 and IMX900, respectively, in which ALD6 was deleted. Counter selection of pUDR240 from IMX901 yielded strain IMX1039. Strain IMX1142, in which the native $A L D 6$ gene was re-introduced in the $S G A 1$ locus, was obtained by co-transformation of pUDR103 and the SGA1-flanked $A L D 6$ cassette into strain IMX1039. Co-transformation of pUDR119 and the SGA1-flanked ALD6 cassette into strain IMX900, following pUDR119 counter selection, yielded strain IMX1120. The empty-vector reference strain IME324 was obtained by transformation of IMX581 with p426-TEF.

\section{Bioreactor batch cultivation}

Anaerobic batch cultures were grown in 2-L bioreactors (Applikon, Schiedam, The Netherlands) on synthetic medium [47] supplemented with acetic acid $\left(3 \mathrm{~g} \mathrm{~L}^{-1}\right.$ final concentration). In high-osmolarity cultures of the acetateconsuming strains IMX776, IMX901 and IMX1142, the concentration of acetic acid was re-set to $3 \mathrm{~g} \mathrm{~L}^{-1}$ when it reached a value below $1.5 \mathrm{~g} \mathrm{~L}^{-1}$, with the addition of glacial acetic acid, to prevent acetic-acid limitation [43]. After autoclaving the mineral salt components of the synthetic medium and acetic acid at $120{ }^{\circ} \mathrm{C}$ for $20 \mathrm{~min}$, anaerobic growth media were supplemented with sterile antifoam C $\left(0.2 \mathrm{~g} \mathrm{~L}^{-1}\right)$ (Sigma-Aldrich), ergosterol $\left(10 \mathrm{mg} \mathrm{L}^{-1}\right)$, Tween $80\left(420 \mathrm{mg} \mathrm{L}^{-1}\right)$ and filter-sterilized vitamin solution [47]. Glucose solutions were autoclaved separately at $110{ }^{\circ} \mathrm{C}$ for $20 \mathrm{~min}$ and added to low- and high-osmolarity media at final concentrations of 20 and $180 \mathrm{~g} \mathrm{~L}^{-1}$ $(1 \mathrm{M})$, respectively. Shake-flask cultures $(100 \mathrm{~mL})$ were inoculated with frozen glycerol stock cultures $(1 \mathrm{~mL})$ and grown on synthetic medium supplemented with glucose (20 $\mathrm{g} \mathrm{L}^{-1}$ final concentration). Samples from these cultures were used as inocula for $100 \mathrm{~mL}$ shake-flask precultures on the same medium, yielding an initial $\mathrm{OD}_{660}$ of 0.1-0.3. Upon reaching mid-exponential phase $\left(\mathrm{OD}_{660}\right.$ 4-6), samples from these pre-cultures were used to inoculate anaerobic bioreactor cultures, yielding an initial $\mathrm{OD}_{660}$ of 0.15-0.2. Anaerobic conditions were maintained by continuously sparging nitrogen gas $(<10 \mathrm{ppm}$ oxygen) at a rate of $0.5 \mathrm{~L} \mathrm{~min}^{-1}$. Norprene tubing and Viton 
O-rings were used to minimize oxygen diffusion into the reactors. In low-osmolarity cultures, the culture $\mathrm{pH}$ was automatically controlled at 5.0 by addition of $2 \mathrm{M} \mathrm{KOH}$. In high-osmolarity cultures ( $\mathrm{pH} 5.0), 12.5 \% \mathrm{v} \mathrm{v}^{-1} \mathrm{NH}_{4} \mathrm{OH}$ solution was used as titrant to prevent nitrogen limitation. The stirrer speed was set at $800 \mathrm{rpm}$, and temperature was controlled at $30^{\circ} \mathrm{C}$. Evaporation was minimized by cooling the outlet gas to $4{ }^{\circ} \mathrm{C}$ in a condenser.

\section{Enzyme-activity assays}

Cell extracts were prepared by sonication [52] of biomass from exponential-phase shake-flask cultures $\left(\mathrm{OD}_{660}\right.$ 5-6) grown on synthetic medium (20 g L $\mathrm{L}^{-1}$ glucose) in an aerobic incubator. Enzyme-activity assays were performed at $30{ }^{\circ} \mathrm{C}$ by continuous spectrophotometric monitoring of the conversion of $\mathrm{NAD}(\mathrm{P}) \mathrm{H}$ to $\mathrm{NAD}(\mathrm{P})^{+}$ at $340 \mathrm{~nm}$. For the determination of acetylating-acetaldehyde dehydrogenase activity [25], cells were sonicated in $100 \mathrm{mM}$ potassium-phosphate buffer (KPB, pH 7.5) with $2 \mathrm{mM} \mathrm{MgCl}$ and $1 \mathrm{mM}$ dithiothreitol. The $1-\mathrm{mL}$ reaction mixture contained $50 \mathrm{mM} \mathrm{KPB} \mathrm{(pH} \mathrm{7.5),}$ $0.15 \mathrm{mM} \mathrm{NADH}$ and 50 or $70 \mu \mathrm{L}$ cell extract. Reactions were started by addition of acetyl-CoA to a final concentration of $0.5 \mathrm{mM}$. To assess if expression of A. fulgidus gpsA resulted in a change in the cofactor preference of glycerol-3-phosphate dehydrogenase (G3PDH) in S. cerevisiae, G3PDH activities were measured by means of a modified version of a published assay optimized for GpsA [45]. In the modified assay, $20 \mathrm{mM}$ Tris- $\mathrm{HCl}(\mathrm{pH}$ 8.2) buffer supplemented with $10 \mathrm{mM}$ EDTA was used for harvesting and storage of cells, and sonication was done in $20 \mathrm{mM}$ Tris- $\mathrm{HCl}$ ( $\mathrm{pH} 8.2$ ) buffer with $2 \mathrm{mM}$ EDTA. The $1-\mathrm{mL}$ reaction mixture contained $50 \mathrm{mM}$ Tris- $\mathrm{HCl}$ (pH 6.6), $2 \mathrm{mM}$ EDTA, $0.15 \mathrm{mM}$ NADH or $\mathrm{NADPH}$ and 50 or $70 \mu \mathrm{L}$ cell extract. The reaction was started by addition of dihydroxyacetone phosphate to a final concentration of $4 \mathrm{mM}$. All assays were performed on samples from two independent cultures, and enzyme activities were proportional to the volume of cell extract added to the assay.

\section{Intracellular glycerol determination}

Shake-flask pre-cultures on synthetic medium $\left(20 \mathrm{~g} \mathrm{~L}^{-1}\right.$ glucose) were inoculated from frozen stocks. After reaching mid-exponential phase, cells were washed with sterile demineralized water and used as inoculum for anaerobic shake-flask cultures on the same medium as the high-osmolarity bioreactor batch cultivations. Anaerobic shake-flask cultures were grown in a Bactron anaerobic chamber (Sheldon Manufacturing, Cornelius, OR) at $30^{\circ} \mathrm{C}$. Mid-exponential-phase cultures were harvested and centrifuged at $4000 \times g$ for $5 \mathrm{~min}$. The supernatant was discarded, and the cells were resuspended in
$0.005 \mathrm{~mol} \mathrm{~L}^{-1} \mathrm{H}_{2} \mathrm{SO}_{4}$ and incubated at $100{ }^{\circ} \mathrm{C}$ for $5 \mathrm{~min}$. The cell suspension was centrifuged at $4000 \times g$ for $5 \mathrm{~min}$, and the supernatant was used for HPLC analysis. For the calculation of the pellet volume, an average density of the pellet of $1.1 \mathrm{~g} \mathrm{~mL}^{-1}$ was used [53]. For the conversion of intracellular glycerol concentration from $g$ ( $g$ dry weight $)^{-1}$ to $\mathrm{g} \mathrm{L}^{-1}$, an intracellular volume of $2.6 \mathrm{~mL}$ (g dry weight $)^{-1}$ was used [54].

\section{Analytical methods}

Biomass dry weight determination, HPLC analysis of extracellular metabolites and correction for ethanol evaporation were performed as previously described [25]. Culture offgas composition was analysed as previously described [25], except for batch cultures grown under high-osmolarity conditions with strains IMX992, IMX884, IMX776 and IMX901, in which production of $\mathrm{CO}_{2}$ was calculated from ethanol production, assuming formation of $1 \mathrm{~mol} \mathrm{CO}$ per mol ethanol produced. Prior to glucose and ethanol concentration measurements in high-osmolarity fermentations, culture supernatant was diluted 1:1 with demineralized water. Product yields and ratios in batch cultures were calculated from a minimum of five samples taken during the mid-exponential growth phase [29]. Biomass concentrations corresponding to samples taken before the mid-exponential growth phase $\left(\mathrm{OD}_{660}<1\right)$ were calculated based on $\mathrm{OD}_{660}$ measurements, using calibration curves based on a minimum of five samples taken in mid-exponential phase for which biomass dry weight and $\mathrm{OD}_{660}$ were measured [29].

\section{Results}

Limited impact of the expression of an acetate-reduction pathway in GPD1 GPD2 S. cerevisiae

Previous studies on acetate reduction by anaerobic, glucose-grown $S$. cerevisiae cultures were based on gpd1 $1 \Delta$ gpd $2 \Delta$ strains $[25,29]$. In these strains, the role of the native glycerol pathway in NADH reoxidation was entirely replaced by reduction of externally supplied acetate to ethanol. To investigate the impact of co-expressing an acetate-reduction pathway with a fully functional glycerol pathway, growth and product formation of strain IMX992 (GPD1 GPD2 sga1::eutE) were analysed in anaerobic, glucose-grown bioreactor batch cultures on $20 \mathrm{~g} \mathrm{~L}^{-1}$ glucose, supplemented with $3 \mathrm{~g} \mathrm{~L}^{-1}$ acetic acid (Fig. 1; Table 3) and compared with the acetate non-reducing reference strain IME324. Under these conditions and consistent with previous reports [29, 43], IME324 (GPD1 GPD2) showed an acetate consumption of $2.43 \mathrm{mmol}$ (g biomass) ${ }^{-1}$ (Table 3 ). In acetate non-reducing strains, consumption of small amounts of acetate can reflect intracellular accumulation and/or use of extracellular-acetate-derived acetyl-CoA as a biosynthetic precursor [55]. Strain IMX992 (GPD1 GPD2 

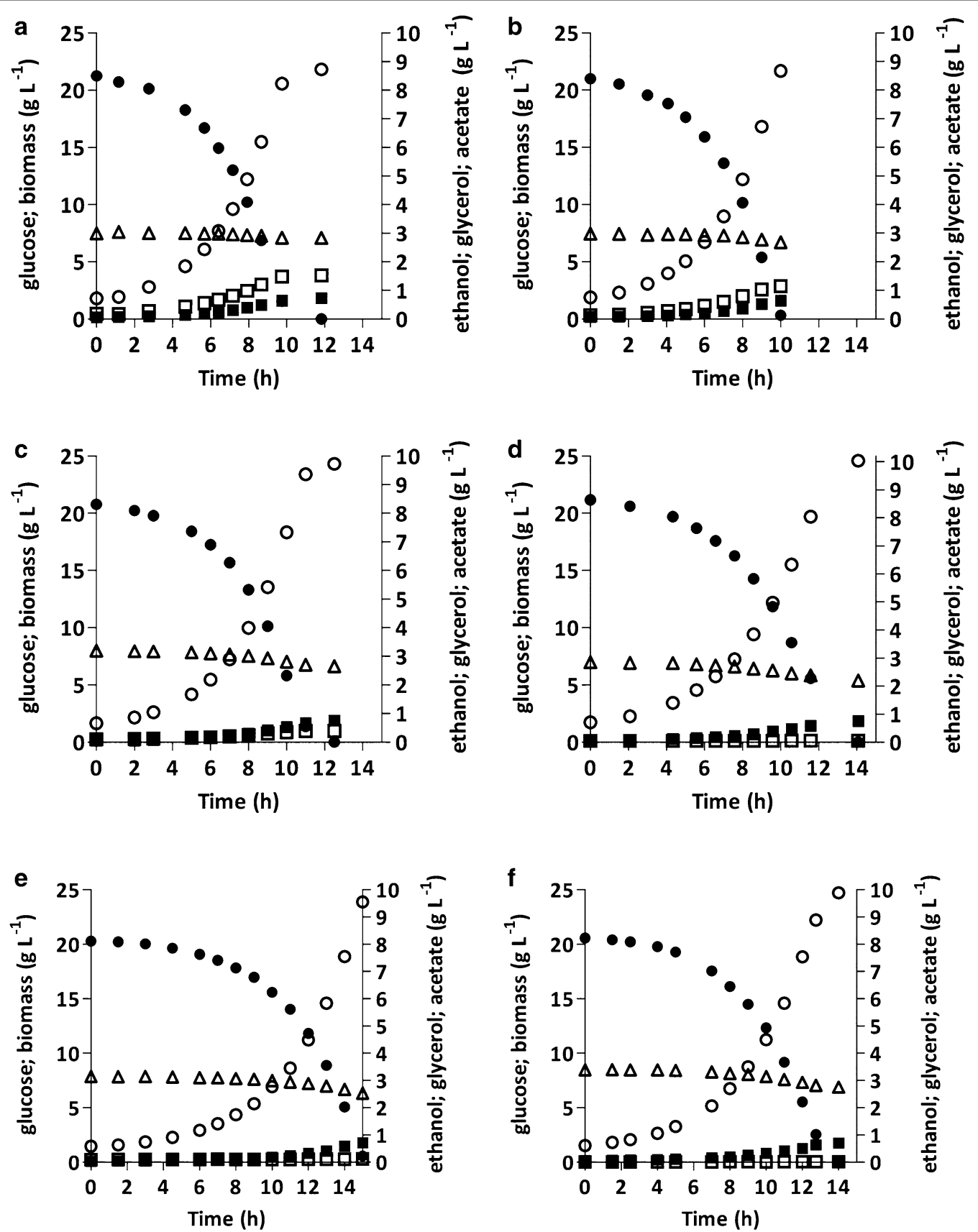

Fig. 1 Growth, glucose consumption and product formation in anaerobic bioreactor batch cultures of S. cerevisiae strains with different genetic modifications in glycerol and acetate metabolism. Cultures were grown on synthetic medium containing $20 \mathrm{~g} \mathrm{~L}^{-1}$ glucose and $3 \mathrm{~g} \mathrm{~L}^{-1}$ acetic acid (pH 5). a strain IME324 (GPD1 GPD2); b strain IMX992 (GPD1 GPD2 sga1::eutE); c strain IMX884 (GPD1 gpd2::eutE); d strain IMX776 (gpd1::gpsA

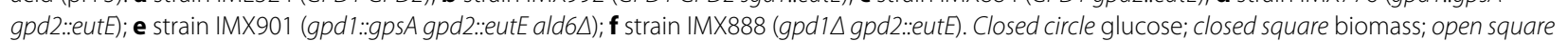
glycerol; open circle ethanol; open triangle acetate. a-f display single representative cultures from a set of two independent duplicate cultures for each strain. Data on strain IMX888 were taken from [29]

sga1::eutE) showed an acetate consumption of $3.35 \mathrm{mmol}$ (g biomass) $^{-1}$, which was only $0.92 \mathrm{mmol}$ (g biomass) $^{-1}$ higher than the acetate consumption by the GPD1 GPD2 reference strain. Conversely, under identical conditions, strain IMX888 (gpd1A gpd2::eutE) showed an acetate consumption of $6.92 \mathrm{mmol}(\mathrm{g} \text { biomass })^{-1}$ in a previous study [29]. Consistent with its marginally higher acetate consumption, glycerol production by strain IMX992 decreased only slightly, from 9.19 to $8.28 \mathrm{mmol}$ glycerol (g biomass) $)^{-1}$, relative to strain IME324 (Table 3). Clearly, in 
Table 3 Specific growth rates $(\mu)$ and stoichiometric relationships between glycerol production and biomass formation, acetate consumption and glucose consumption, and acetate consumption and biomass formation in anaerobic bioreactor batch cultures of $S$. cerevisiae strains with different genetic modifications in glycerol and acetate metabolism

\begin{tabular}{|c|c|c|c|c|c|c|}
\hline Strain & IME324 & IMX992 & IMX884 & IMX776 & IMX901 & IMX888 \\
\hline Relevant genotype & $\begin{array}{l}\text { GPD1 } \\
\text { GPD2 }\end{array}$ & $\begin{array}{l}\text { GPD1 } \\
\text { GPD2 } \\
\text { sga1::eutE }\end{array}$ & $\begin{array}{l}\text { GPD1 } \\
\text { gpd2::eutE }\end{array}$ & $\begin{array}{l}\text { gpd1:::gpsA } \\
\text { gpd2::eutE }\end{array}$ & $\begin{array}{l}\text { gpd1:::gpsA } \\
\text { gpd2::eutE } \\
\text { ald6 } 6 \Delta\end{array}$ & $\begin{array}{l}\text { gpd1 } 1 \Delta \\
\text { gpd2::eutE }\end{array}$ \\
\hline$\mu\left(\mathrm{h}^{-1}\right)$ & $0.31 \pm 0.01$ & $0.30 \pm 0.00$ & $0.31 \pm 0.01$ & $0.24 \pm 0.01$ & $0.24 \pm 0.01$ & $0.26 \pm 0.01$ \\
\hline Ratio glycerol produced/biomass [mmol ( $\mathrm{g}$ biomass) $\left.{ }^{-1}\right]$ & $9.19 \pm 0.08$ & $8.28 \pm 0.14$ & $1.92 \pm 0.06$ & $<0.1$ & $<0.1$ & $<0.1$ \\
\hline Ratio acetate consumed/biomass [mmol (g biomass) $\left.{ }^{-1}\right]$ & $2.43 \pm 0.16$ & $3.35 \pm 0.08$ & $5.77 \pm 0.25$ & $6.66 \pm 0.01$ & $6.41 \pm 0.28$ & $6.92 \pm 0.12$ \\
\hline Ratio acetate consumed/glucose $\left(\mathrm{g} \mathrm{g}^{-1}\right)$ & $0.010 \pm 0.000$ & $0.015 \pm 0.000$ & $0.026 \pm 0.001$ & $0.031 \pm 0.001$ & $0.031 \pm 0.000$ & $0.032 \pm 0.000$ \\
\hline
\end{tabular}

Cultures were grown on synthetic medium containing $20 \mathrm{~g} \mathrm{~L}^{-1}$ glucose and $3 \mathrm{~g} \mathrm{~L}^{-1}$ acetic acid (pH 5). Specific growth rates and stoichiometries were calculated from the mid-exponential growth phase and represent averages \pm mean deviations of data obtained from independent duplicate cultures. In all cultures, carbon recoveries were between 95 and 100\%. Enzyme activities of acetylating-acetaldehyde dehydrogenase in cell extracts of eutE-expressing strains were similar (Additional file 2)

a Data on strain IMX888 were taken from [29]

glucose-fermenting engineered S. cerevisiae strains, EutEbased acetate reduction could not efficiently compete for $\mathrm{NADH}$ with a fully functional native glycerol pathway.

\section{Deletion of GPD2 improves acetate reduction by an eutE-expressing strain}

GPD2 encodes the redox-regulated isoenzyme of G3PDH and its deletion has been reported to cause impaired anaerobic growth of S. cerevisiae [23, 35]. In acetate-supplemented anaerobic cultures of strain IMX884 (GPD1 gpd2::eutE), eutE expression fully compensated for the absence of a functional Gpd2 enzyme, both in terms of specific growth rate and in terms of biomass yield on glucose (Table 3; Fig. 1; Additional file 3). Compared to strain IMX992 (GPD1 GPD2 sga1::eutE), strain IMX884 showed a fourfold lower production of glycerol [1.92 and $8.28 \mathrm{mmol}$ glycerol (g biomass) ${ }^{-1}$, respectively] and a correspondingly higher EutE-based acetate consumption [3.34 and $0.92 \mathrm{mmol}$ acetate (g biomass) $)^{-1}$, respectively], corrected for acetate consumption by the acetate non-reducing reference strain IME324, resulting in an ethanol yield on glucose of $0.46 \mathrm{~g} \mathrm{~g}^{-1}$ (Additional file 3). These results indicate that, at least in low-osmolarity media, inactivation of GPD2 enables the EutE-based acetate-reduction pathway to efficiently compete for redox equivalents with the glycerol pathway. This engineering strategy not only resulted in a markedly higher acetate consumption, but also in a higher ethanol yield on glucose than observed in the acetate non- reducing reference strain IME324 (Table 3; Additional file 3).

\section{Functional expression of an NADPH-preferring G3PDH in S. cerevisiae}

As outlined above, expression of the $\mathrm{NADP}^{+}$-preferring G3PDH encoded by $A$. fulgidus gpsA might enable strategies to uncouple the roles of glycerol metabolism in yeast osmotolerance and redox balancing. To investigate whether gpsA can be functionally expressed in S. cerevisiae, its coding sequence was codon optimized for expression in yeast and integrated at the GPD1 locus of strain IMX581 (along with integration of eutE at the GPD2 locus), yielding strain IMX776 (gpd1::gpsA gpd2::eutE). This insertion was designed to place gpsA under the control of the GPD1 promoter and terminator, in order to enable upregulation of its expression at high-osmolarity [20,21].

Enzyme-activity assays in cell extracts showed that, in strain IMX776, replacement of the native GPD1 and GPD2 genes by $g p s A$ resulted in a switch in cofactor preference of glycerol-3-phosphate dehydrogenase (G3PDH, Fig. 2). The gpsA-expressing strain showed in vitro activities of $0.103 \pm 0.004$ and $0.006 \mu \mathrm{mol} \mathrm{mg}$ protein ${ }^{-1} \mathrm{~min}^{-1}$ with NADPH and NADH, respectively. As a result, the ratio of NADPH- and NADH-linked rates of dihydroxyacetone phosphate reduction was ca. 500-fold higher in strain IMX776 than in the reference strain IMX992, which expresses the native GPD1 and GPD2 genes. These observations are consistent with a previous report on the cofactor preference of GpsA, expressed in E. coli [45], and show that the enzyme was functionally expressed in yeast.

\section{Increased acetate reduction and decreased glycerol} production in a gpsA-expressing yeast strain

The combined activity of acetyl-CoA synthetase and EutE, both of which are essential for acetate reduction and Ald6, the major $\mathrm{NADP}^{+}$-dependent, cytosolic isoform of acetaldehyde dehydrogenase [56], could potentially form an ATP-driven dehydrogenase cycle $\left(\mathrm{NADH}+\mathrm{NADP}^{+} \rightarrow \mathrm{NADPH}+\mathrm{NAD}^{+}[29]\right)$. In $g p s A-$ expressing strains, NADPH formed via this cycle might increase glycerol production and, consequently, decrease ethanol yields (Additional file 8). Therefore, ALD6 was deleted in the gpsA-expressing, acetate-reducing strain IMX776 (gpd1::gpsA gpd2::eutE), yielding strain IMX901. 


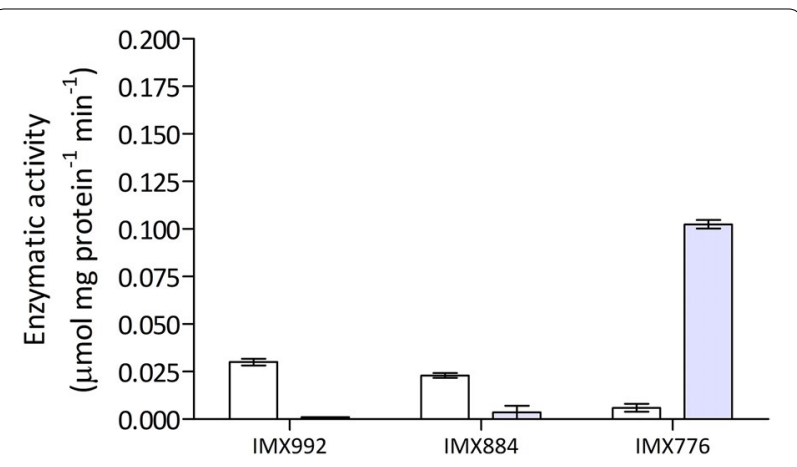

Fig. 2 Specific rates of NADH-dependent (white bars) and NADPHdependent (blue bars) reduction of dihydroxyacetone phosphate by cell extracts of shake-flask cultures on synthetic medium $\left(20 \mathrm{~g} \mathrm{~L}^{-1}\right.$ glucose) of S. cerevisiae strains IMX992 (GPD1 GPD2), IMX884 (GPD1 gpd2::eutE) and IMX776 (gpd1::gpsA gpd2::eutE). Data represent averages \pm mean deviations of assays on independent duplicate cultures

In anaerobic, acetate-supplemented bioreactor batch cultures, the specific growth rate of strain IMX776 (gpd1::gpsA gpd2::eutE) was $0.24 \mathrm{~h}^{-1}$, which was ca. $20 \%$ lower than that of the reference strain IME324 (GPD1 GPD2). The physiology of strain IMX776 in these anaerobic low-osmolarity cultures, including the stoichiometry of biomass formation and acetate consumption, closely resembled that of strain IMX888 (gpd1 gpd2::eutE) (Table 3; Fig. 1; Additional file 3). Virtually no extracellular glycerol was formed by strain IMX776, indicating that, under these conditions, the in vivo activity of NADPHdependent glycerol production in this strain was minimal. Consistent with this notion, growth and product formation in anaerobic cultures of strain IMX901 (gpd1::gpsA gpd2::eutE ald6 4 ) was similar to the observed performance of strains IMX776 or IMX888 under these conditions.

\section{Growth at high-osmolarity negatively affects acetate reduction by a $g p d 2 \Delta$ strain}

To assess the impact of high-osmolarity on the acetate reduction observed in the GPD1 gpd2:: eutE strain IMX884, its performance was compared with that of strain IMX992 (GPD1 GPD2 sga1::eutE) in anaerobic bioreactor batch cultures grown on $1 \mathrm{~mol} \mathrm{~L}^{-1}\left(180 \mathrm{~g} \mathrm{~L}^{-1}\right)$ glucose. In contrast to the low-osmolarity cultures, in which strains continued to grow exponentially until glucose was depleted (Fig. 1), high-osmolarity conditions showed a biphasic growth profile, in which the exponential phase was followed by second, slower growth phase (Fig. 3). This biphasic growth profile probably reflects a nutritional limitation other than carbon source depletion. For example, concentrations of the anaerobic growth factors Tween- 80 and ergosterol were not increased in high-osmolarity media to avoid potential toxic effects [15]. A similar growth pattern in high-glucose cultures has been reported previously [43].
Consistent with previously reported data on a congenic, non-acetate-reducing GPD1 GPD2 strain grown under high-osmolarity conditions [43], the initial specific growth rate of strain IMX992 (GPD1 GPD2 sga1::eutE) was not affected by increasing the glucose concentration in the medium to $1 \mathrm{~mol} \mathrm{~L}^{-1}$ (Tables 3,4 ). Acetate consumption in the high-osmolarity cultures by this strain was even slightly lower than observed during growth on $20 \mathrm{~g} \mathrm{~L}^{-1}$ glucose $\left[2.67\right.$ and $3.35 \mathrm{mmol}\left(\mathrm{g} \mathrm{biomass}^{-1}\right.$ ), respectively]. This observation indicates that, also under high-osmolarity conditions, EutE-mediated acetate reduction could not efficiently compete for $\mathrm{NADH}$ with a fully functional glycerol pathway.

Strain IMX884 (GPD1 gpd2::eutE) showed a 10\% lower specific growth rate in high-osmolarity medium than in cultures grown on a low glucose concentration (Tables 3, 4). This, only minor, difference is consistent with the reported predominant role of the Gpd1 isoenzyme in osmoregulation [20, 21, 57]. Relative to its performance in low-osmolarity cultures, growth on $1 \mathrm{~mol} \mathrm{~L}^{-1}$ glucose led to a threefold increase in extracellular glycerol production [(6.34 mmol (g biomass) ${ }^{-1}$ versus $1.92 \mathrm{mmol}$ (g biomass $)^{-1}$ and a corresponding decrease in acetate consumption $\left[2.98 \mathrm{mmol}\right.$ (g biomass) ${ }^{-1}$ versus $5.77 \mathrm{mmol}$ (g biomass $)^{-1}$ ] (Tables 3,4$)$. These changes largely eliminated the fourfold difference in glycerol production between strains IMX992 and IMX884 that was observed in low-osmolarity cultures (Tables 3, 4). After complete glucose consumption, concentrations of acetic acid, glycerol and ethanol reached similar concentrations in high-osmolarity cultures of the two strains (Fig. 3). These results indicate that, even when GPD2 is deleted, highosmolarity conditions impeded the competition of the EutE-based acetate-reduction pathway for NADH with the glycerol pathway, possibly due to osmotic-stressinduced upregulation of GPD1.

\section{Replacement of GPD1 and GPD2 by gpsA uncouples the roles of glycerol formation in redox metabolism and osmoregulation}

To test whether replacement of the yeast $\mathrm{NAD}^{+}$-dependent Gpd isoenzymes by an $\mathrm{NADP}^{+}$-preferring $\mathrm{G} 3 \mathrm{PDH}$ can uncouple the roles of glycerol formation in osmoregulation and redox metabolism, the growth and product formation of strain IMX776 (gpd1::gpsA gpd2::eutE) were investigated in high-osmolarity cultures. In contrast to strains IMX992 and IMX884, strain IMX776 showed a lag phase of ca. $50 \mathrm{~h}$ under these conditions (Fig. 3; Additional file 4), and its specific growth rate was $60 \%$ lower than that in low-osmolarity cultures (Tables 3,4 ). While, under lowosmolarity conditions, this strain did not produce extracellular glycerol, high-osmolarity batch cultures showed a glycerol production of $3.29 \mathrm{mmol}$ (g biomass) ${ }^{-1}$ (Table 4). 

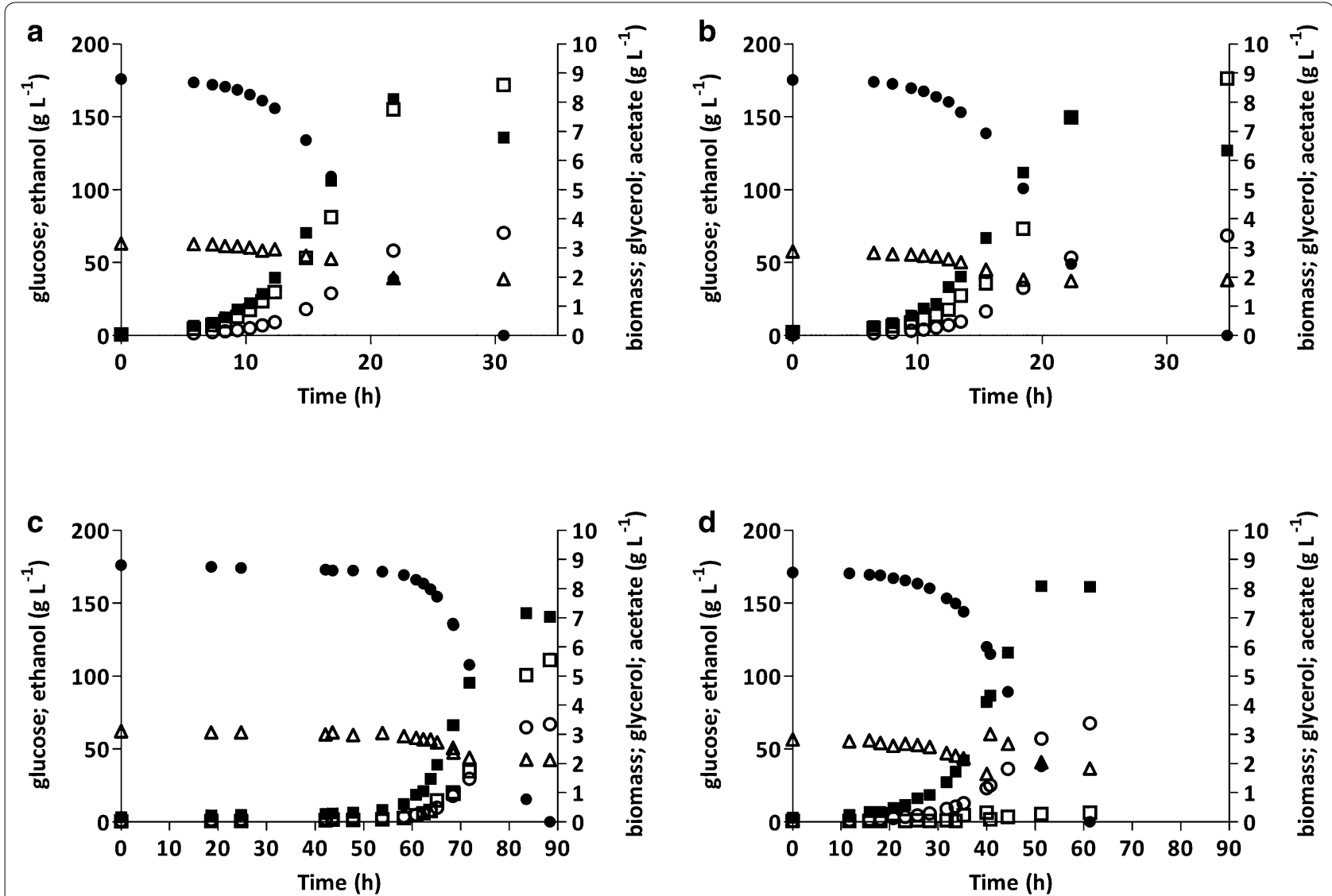

Fig. 3 Growth, glucose consumption and product formation in anaerobic bioreactor batch cultures of S. cerevisiae strains with different genetic modifications in glycerol and acetate metabolism. Cultures were grown on synthetic medium containing $180 \mathrm{~g} \mathrm{~L}^{-1} \mathrm{glucose}$ and $3 \mathrm{~g} \mathrm{~L}^{-1}$ acetic acid (pH 5). a Strain IMX992 (GPD1 GPD2 sga1:::eutE); b strain IMX884 (GPD1 gpd2:::eutE); c strain IMX776 (gpd1::gpsA gpd2::eutE); d strain IMX901 (gpd1::gpsA gpd2::eutE ald6 $\triangle$ ). Closed circle glucose; closed square biomass; open square glycerol; open circle ethanol; open triangle acetate. a-c display single representative cultures from a set of two independent duplicate cultures for each strain. In the culture of IMX901, acetic acid was added externally immediately after the exponential growth phase was finished

Table 4 Specific growth rates $(\mu)$, yields $(Y)$ of biomass, ethanol and glycerol on glucose and stoichiometric relationships between glycerol production and biomass formation, acetate consumption and glucose consumption, and acetate consumption and biomass formation in anaerobic bioreactor batch cultures of $S$. cerevisiae strains with different genetic modifications in glycerol and acetate metabolism

\begin{tabular}{|c|c|c|c|c|}
\hline Strain & IMX992 & IMX884 & IMX776 & IMX901 \\
\hline Relevant genotype & $\begin{array}{l}\text { GPD1 GPD2 } \\
\text { sga1::eutE }\end{array}$ & $\begin{array}{l}\text { GPD1 } \\
\text { gpd2::eutE }\end{array}$ & $\begin{array}{l}\text { gpd1:::gpsA } \\
\text { gpd2::eutE }\end{array}$ & $\begin{array}{l}\text { gpd1:::gpsA } \\
\text { gpd2::eutE } \\
\text { ald6 } 6\end{array}$ \\
\hline$\mu\left(h^{-1}\right)$ & $0.28 \pm 0.02$ & $0.27 \pm 0.00$ & $0.14 \pm 0.00$ & $0.12 \pm 0.02$ \\
\hline$Y$ biomass/glucose $\left(\mathrm{g} \mathrm{g}^{-1}\right)$ & $0.087 \pm 0.001$ & $0.085 \pm 0.000$ & $0.089 \pm 0.000$ & $0.077 \pm 0.013$ \\
\hline Y ethanol/glucose $\left(\mathrm{g} \mathrm{g}^{-1}\right)$ & $0.43 \pm 0.01$ & $0.42 \pm 0.02$ & $0.47 \pm 0.01$ & $0.49 \pm 0.00$ \\
\hline Y glycerol/glucose $\left(\mathrm{g} \mathrm{g}^{-1}\right)$ & $0.07 \pm 0.00$ & $0.05 \pm 0.00$ & $0.02 \pm 0.00$ & $<0.001$ \\
\hline Glycerol produced/biomass [mmol (g biomass) $\left.{ }^{-1}\right]$ & $8.76 \pm 0.25$ & $6.34 \pm 0.26$ & $3.29 \pm 0.41$ & $<0.1$ \\
\hline Acetate consumed/biomass [mmol ( $\mathrm{g}$ biomass) $\left.{ }^{-1}\right]$ & $2.67 \pm 0.96$ & $2.98 \pm 0.08$ & $2.88 \pm 0.17$ & $5.71 \pm 0.15$ \\
\hline Acetate consumed/glucose $\left(\mathrm{g} \mathrm{g}^{-1}\right)$ & $0.011 \pm 0.001$ & $0.015 \pm 0.000$ & $0.016 \pm 0.000$ & $0.027 \pm 0.003$ \\
\hline
\end{tabular}

Cultures were grown on synthetic medium containing $180 \mathrm{~g} \mathrm{~L}^{-1}$ glucose and $3 \mathrm{~g} \mathrm{~L}^{-1}$ acetic acid (pH 5). Specific growth rates and stoichiometries were calculated from the mid-exponential growth phase and represent averages \pm mean deviations of data obtained from independent duplicate cultures 
After glucose depletion, the glycerol concentration in high-osmolarity cultures of strain IMX776 was 44\% lower than observed for strain IMX992 (GPD1 GPD2 sga1::eutE) (Fig. 3).

Strain IMX776 showed a much lower acetate consumption in the high-glucose cultures than in lowosmolarity cultures (Tables 3,4). This difference could be caused by an increased flux through the cytosolic, $\mathrm{NADP}^{+}$-dependent acetaldehyde dehydrogenase Ald6, coupled to the increased demand for NADPH in the cytosolic GpsA reaction. Generating NADPH via the oxidation of acetaldehyde to acetate, which can subsequently be reduced to ethanol via acetyl-CoA synthetase, EutE and $\mathrm{NAD}^{+}$-dependent alcohol dehydrogenase, would result in less extracellular acetate being consumed for NADH reoxidation (Additional file 8). An increased production of acetate has been previously observed upon an increase of cytosolic NADPH demand in anaerobic $S$. cerevisiae cultures [29].

Consistent with the hypothesis outlined above, deletion of $A L D 6$ had a strong impact on the physiology of anaerobic cultures of acetate-reducing gpsA-expressing $S$. cerevisiae. Although the specific growth rates of strain IMX776 (gpd1::gpsA gpd2:eutE) and strain IMX901 (gpd1::gpsA gpd2:eutE ald64) in high-osmolarity cultures were similar (Table 4), complete absence of a lag phase decreased the overall fermentation time of the latter strain by ca. 35 h (Fig. 3; Additional file 4). In addition, strain IMX901 fully relied on exogenous acetic acid supply for its redox balancing. When, after exponential growth was finished, no additional acetate was provided, growth and glucose consumption slowed down considerably (Additional file 5). A similar addition of acetate to a high-osmolarity batch culture of strain IMX776 did not affect its growth (Additional file 5).

In contrast to strains IMX884 and IMX776, strain IMX901 retained a glycerol non-producing phenotype throughout growth in bioreactor cultures on high-osmolarity medium, resulting in a 13\% higher ethanol yield on glucose compared to strain IMX992 (GPD1 GPD2 sga1::eutE; Table 4). This, in combination with a measured intracellular glycerol concentration of $5.3 \pm 0.04 \mathrm{~g} \mathrm{~L}^{-1}$ in anaerobic shake-flask cultures of strain IMX901 on high-osmolarity medium, indicated a complete intracellular retention of glycerol formed via GpsA in this strain. When additional acetate was added to high-osmolarity bioreactor cultures of strain IMX901 immediately after the exponential phase, no extracellular glycerol was detectable (Fig. 3). However, when acetate was added $20 \mathrm{~h}$ into the stationary phase (Additional file 5), low concentrations of glycerol were detectable $\left(<1 \mathrm{~g} \mathrm{~L}^{-1}\right.$ final concentration).

\section{Growth of an acetate-reducing gpd $1 \Delta$ gpd $2 \Delta$ strain in high-osmolarity medium}

In the experiments discussed above, the glycerol nonproducing, acetate-reducing strain IMX888 (gpd1A gpd2::eutE) was included as a reference strain. Surprisingly, despite the absence of a functional glycerol pathway, this strain consistently grew in anaerobic high-osmolarity cultures, after a lag phase of ca. $75 \mathrm{~h}$ (Additional file 6). Furthermore, the strain retained its acetate-reducing phenotype, with minimal concentrations of acetate and glycerol having been produced upon glucose depletion (Additional file 7). This result contradicts earlier reports of a complete inability of gpd1 $\operatorname{gpd} 2 \Delta$ strains to grow at high-osmolarity $[20,21,43]$. We therefore re-tested growth of strain IMZ160 ( $g p d 1 \Delta g p d \Delta m h p F)$ [43], which has been previously reported not to grow under the highosmolarity conditions used in the present study. Consistent with previous observations, this strain failed to grow in high-osmolarity cultures, even after $300 \mathrm{~h}$ of incubation (Additional file 6). The different phenotypes of two acetylating-acetaldehyde expressing, glycerol-negative S. cerevisiae strains may reflect the lower in vivo activities of heterologously expressed MhpF relative to EutE $[25,29]$. To investigate a possible involvement of $A L D 6$ (see above and Additional file 8), this gene was deleted in strain IMX888, yielding strain IMX900 (gpd1 gpd2::eutE ald6 6 ). The latter strain showed a strongly reduced lag phase in high-osmolarity medium relative to its parental strain IMX888, thereby reducing the total fermentation time by ca. $45 \mathrm{~h}$ (Additional file 6). However, the overall fermentation time of strain IMX900 was still considerably longer than that of the gpsA-expressing ald $6 \Delta$ strain IMX901 (Additional file 6; Fig. 3). Similar to its parental strain, IMX900 retained its acetate-reducing phenotype and produced only trace amounts of extracellular glycerol (Additional file 7). When the native S. cerevisiae ALD6 gene, including its promoter and terminator sequences, was integrated at the SGA1 locus of strains IMX900 and IMX901, the resulting strains (IMX1120 and IMX1142) again showed a prolonged lag phase, confirming the detrimental effect of Ald6 in this experimental context (Additional file 6; Fig. 3).

\section{Discussion}

Expression of a heterologous acetylating-acetaldehyde dehydrogenase (A-ALD) can fully restore anaerobic growth in acetate-supplemented cultures of $S$. cerevisiae strains that lack a functional glycerol pathway $[25,29]$. However, the minor decrease in glycerol formation observed upon A-ALD expression in a GPD1 GPD2 strain (Table 3) indicated that A-ALD-based acetate reduction cannot efficiently compete for NADH with a fully functional, native glycerol pathway. Recently, a 40\% decrease in glycerol yield 
was reported upon A-ALD expression in an industrial $S$. cerevisiae strain [58]. The higher relative impact of A-ALD expression in the industrial strain coincided with a twofold lower glycerol yield relative to that of the laboratory reference strain used in this work. These observations identify reduction of the capacity of the native glycerol pathway as an interesting strategy for facilitating NADH reoxidation via acetate reduction. Indeed, deletion of GPD2, which encodes the major isoenzyme of G3PDH in anaerobic, low-osmolarity cultures of $S$. cerevisiae [21, 23] strongly stimulated acetate reduction (Table 3). By enabling an over fourfold lower glycerol yield and corresponding increase in ethanol production by acetate reduction, without any reduction in specific growth rate, combined deletion of GPD2 and expression of an A-ALD offers a promising strategy for improving ethanol production in low-osmolarity, acetic-acid containing media. In such processes, osmolarity may be limited by fed-batch cultivation regimes and/ or by simultaneous saccharification and fermentation of polymeric feedstocks $[59,60]$. Reducing the capacity of the glycerol pathway may be similarly effective in other metabolic engineering strategies for redirecting $\mathrm{NADH}$ reoxidation in anaerobic yeast cultures, such as the use of $\mathrm{CO}_{2}$ by Calvin-cycle-enzyme expressing yeast cultures [24], and for expression of A-ALD in engineered xylose-consuming S. cerevisiae strains based on expression of heterologous xylose reductase and xylitol dehydrogenase enzymes [26].

Compared to its strong impact under low-osmolarity conditions, deletion of GPD2 had a much smaller effect on glycerol production and acetate reduction in cultures of A-ALD-expressing S. cerevisiae grown on $1 \mathrm{~mol} \mathrm{~L}^{-1}$ glucose (Table 4). This difference probably reflects the extensively documented, strong upregulation of GPD1 under hyperosmotic stress [20,61, 62], which is at least partly controlled by the Hog1 MAP-kinase cascade $[20,63]$. Together with the increased intracellular glycerol retention [17, 64], upregulation of G3PDH activity plays a key role in the yeast osmotic stress response. The dual role of G3PDH enzymes in redox homeostasis and osmotolerance represents a challenge in redirecting $\mathrm{NADH}$ reoxidation in high-osmolarity, anaerobic yeast cultures towards acetate reduction. The results presented here provide a proof of principle for separating the roles of glycerol production in NADH reoxidation and osmotolerance by exchanging the native $\mathrm{NAD}^{+}$-dependent G3PDH enzymes for a heterologous, $\mathrm{NADP}^{+}$-preferring enzyme (A. fulgidus GpsA). In contrast to a gpd1 $1 \Delta$ gpd $2 \Delta$ A-ALD-expressing strain, the resulting strain was, after a lag phase, able to grow anaerobically on $1 \mathrm{~mol} \mathrm{~L}^{-1}$ glucose and showed an almost twofold lower glycerol yield than a GPD1 GPD2 reference strain (Table 4). Additional deletion of $A L D 6$ eliminated the lag phase as well as extracellular glycerol production, yielding a strain with stoichiometric acetate consumption and ca. 13\% higher ethanol yield on glucose in high-osmolarity cultures than observed for a GPD1 GPD2 reference strain (Table 4). This ethanol yield improvement was consistent with the results obtained in studies on glucose-grown, acetate-reducing strains that were not further engineered for osmotolerance $[25,29,30]$ and in an osmotolerant acetate-reducing strain obtained after prolonged laboratory evolution [43].

Several factors may explain the strong impact of deleting ALD6 on high-osmolarity cultures. In glucose-grown cultures of wild-type $S$. cerevisiae strains, $\mathrm{NADP}^{+}$-dependent oxidation of acetaldehyde to acetate by Ald6 can account for ca. $20 \%$ of the total cytosolic NADPH requirement $[65,66]$. Even possibly higher contributions of Ald6 have been reported in genetic backgrounds that affect NADPH supply via other pathways [29, 67]. In gpsA-expressing strains, NADPH generated by acetate formation via Ald6 can directly contribute to glycerol production. Since, during growth on glucose, $\mathrm{NADPH}$ formation by Ald6 is coupled to equimolar generation of NADH in glycolysis, the increased acetate formation via Ald6 can help meet an increased NADH demand for glycerol production via Gpd1 and/or Gpd2 during hyper-osmotic stress [20,63]. Furthermore, Ald6, together with yeast acetyl-CoA synthetases (Acs1, Acs2) and heterologously expressed A-ALD, have been proposed to form an ATP-driven transhydrogenase cycle [29]. Activity of such a cycle in high-osmolarity cultures, possibly stimulated by upregulation of $A L D 6$, could impose an ATP drain that impedes growth under the combined stresses of high osmolarity and acetate uncoupling. Elimination of such an ATP drain could explain why the deletion of $A L D 6$ eliminated lag phases in highosmolarity, acetate-supplemented cultures. Increased adaptation phases, reflecting a non-genetic population heterogeneity, are well documented in acetate-stressed $S$. cerevisiae cultures $[68,69]$. Recent studies on engineered A-ALD expressing $S$. cerevisiae strains in low- and medium-osmolarity cultures $[26,29]$ lend further support to the conclusion that deletion of ALD6 is a key step in engineering efficient pathways for acetate reduction in S. cerevisiae.

While a gpd $1 \Delta$ gpd $2 \Delta$ ald $6 \Delta$ strain expressing gpsA and eutE showed an excellent stoichiometry in terms of ethanol yield and acetate reduction, its growth rate in highosmolarity cultures was substantially lower than that of strains expressing GPD1 and/or GPD2. To minimize costs of yeast propagation and to maximize ethanol productivity, a high maximum specific growth rate is important for application in lignocellulosic ethanol production. As previously demonstrated for a $g p d 1 \Delta \operatorname{gpd} 2 \Delta$ strain expressing $m h p F$ [43], evolutionary engineering can enable selection for faster-growing mutants. Alternatively, growth kinetics 
may be improved by optimizing the expression levels of $\mathrm{NADPH}$-dependent G3PDH and/or by improving the availability of cytosolic NADPH $[65,66]$.

In view of the extensively documented, central role of G3PDH in the hyperosmotic-stress response of $S$. cerevisiae $[17,70]$, the slow but reproducible anaerobic growth of a gpd1 $1 \Delta$ gpd $2 \Delta$ ald $6 \Delta$, eutE-expressing S. cerevisiae strain in a medium containing $1 \mathrm{~mol} \mathrm{~L}^{-1}$ glucose was an unexpected result. In addition to possible contributions of the ald $6 \Delta$ mutation and/or eutE expression to osmotolerance, the lag phases of gpd1 $1 \Delta$ gpd $2 \Delta$ strains in high-osmolarity cultures may have obscured this interesting phenotype in previous short-term growth studies $[20,21]$. Osmotolerance in S. cerevisiae is a complex, multi-gene phenotype [71] and, especially upon sudden exposure to osmotic stress, G3PDH-independent mechanisms have been proposed to contribute to osmotolerance [64, 72], such as trehalose accumulation in osmotically challenged cultures growing on galactose [73]. Alternatively, intracellular glycerol could be derived via a G3PDH-independent pathway by de-acylation of acyl-glycerol-3-phosphate, which can be formed from dihydroxyacetone phosphate (DHAP) by the combined activities of DHAP acyltransferase and NADPH-linked 1-acylglycerol-3-phosphate acyltransferase [74]. Activity of the latter pathway may explain low levels of glycerol production in an A-ALD-expressing gpd $1 \Delta$ gpd $2 \Delta$ strain evolved for increased osmotolerance [43]. The acetatereducing, $g p d 1 \Delta g p d 2 \Delta$ strains constructed in this work provide an interesting experimental platform for further fundamental research on G3PDH-independent mechanisms for osmotolerance in S. cerevisiae.

\section{Conclusions}

Deletion of GPD2 provides a straightforward engineering strategy for maximizing the positive impact of A-ALDbased, engineered pathways in low-osmolarity cultures of $S$. cerevisiae, by improving acetate conversion and ethanol yields. Replacement of the $\mathrm{NAD}^{+}$-dependent $S$. cerevisiae glycerol-3P dehydrogenases by a heterologous $\mathrm{NADP}^{+}$-dependent enzyme enables uncoupling of the function of glycerol as an osmoprotectant from its role in cellular redox-cofactor balancing. When combined with a deletion of $A L D 6$, thereby eliminating the influence of cytosolic $\mathrm{NADP}^{+}$-dependent acetaldehyde oxidation on redox metabolism, this engineering strategy enables anaerobic growth and efficient acetate reduction, with its associated improvement of ethanol yields, in high-osmolarity cultures. If the growth kinetics of the resulting strains can be further improved, this approach is highly promising for application in high-gravity processes for conversion of acetate-containing, lignocellulosic hydrolysates.

\section{Additional files}

Additional file 1. Primers used in this study.

Additional file 2. Specific rates of EutE-dependent reduction of acetylCoA by cell extracts of shake-flask cultures on synthetic medium $\left(20 \mathrm{~g} \mathrm{~L}^{-1}\right)$ glucose. From left to right: S. cerevisiae strains IMX992 (GPD1 GPD2 sga1::eutE), IMX884 (GPD1 gpd2::eutE) and IMX776 (gpd1::gpsA gpd2:::eutE). Data represent averages \pm mean deviations of assays on independent duplicate cultures.

Additional file 3. Biomass and product yields in anaerobic bioreactor batch cultures of S. cerevisiae strains with different genetic modifications in glycerol and acetate metabolism. Cultures were grown on synthetic medium containing $20 \mathrm{~g} \mathrm{~L}^{-1}$ glucose and $3 \mathrm{~g} \mathrm{~L}^{-1}$ acetic acid ( $\mathrm{pH} 5$ ). Bars refer to the following engineered S. cerevisiae strains: IME324 (GPD1 GPD2); IMX992 (GPD1 GPD2 sga1:::eutE); IMX884 (GPD1 gpd2::eutE); IMX776 (gpd1::gpsA gpd2::eutE); IMX901 (gpd1:::gpsA gpd2::eutE ald6வ); IMX888 (gpd1 $\triangle g p d 2:: e u t E)$. A, biomass yield on glucose; $B$, ethanol yield on glucose (corrected for ethanol evaporation); C, glycerol yield on glucose. Data represent the averages \pm mean deviations of measurements on independent duplicate cultures for each strain. Data on strain IMX888 were taken from [29].

Additional file 4. Plots of $\ln \left(\mathrm{OD}_{660}\right)$ values versus time in anaerobic bioreactor batch cultures of $\mathrm{S}$. cerevisiae strains with different genetic modifications in glycerol and acetate metabolism (from inoculation to mid-exponential phase). Cultures were grown on synthetic medium containing $180 \mathrm{~g} \mathrm{~L}^{-1}$ glucose and $3 \mathrm{~g} \mathrm{~L}^{-1}$ acetic acid ( $\mathrm{pH}$ 5). $\mathbf{m}$, strain IMX992 (GPD1 GPD2 sga1::eutE); a, strain IMX884 (GPD1 gpd2::eutE); $\diamond$, strain IMX776 (gpd1::gpsA gpd2:::eutE); $\triangle$, strain IMX901 (gpd1:::gpsA gpd2::eutE ald $6 \Delta$ ). The figure shows representative cultures of independent duplicate experiments.

Additional file 5. Growth, glucose consumption and product formation in anaerobic bioreactor batch cultures of $S$. cerevisiae strains with different genetic modifications in glycerol and acetate metabolism. Cultures were grown on synthetic medium containing $180 \mathrm{~g} \mathrm{~L}^{-1}$ glucose and $3 \mathrm{~g} \mathrm{~L}^{-1}$ acetic acid (pH 5). A, strain IMX776 (gpd1:::gpsA gpd2::eutE); B, strain IMX901 (gpd1::.gpsA gpd2::eutE ald6 $\triangle$ ). Symbols: O, glucose; $\mathbf{\square}$, biomass; $\square$, glycerol; $\bigcirc$ ethanol; $\triangle$, acetate. In the case of IMX776, acetic acid was added externally immediately after the exponential growth phase was finished. In the case of IMX901, acetic acid was added externally after $20 \mathrm{~h}$ in stationary phase.

Additional file 6. $\mathrm{CO}_{2}$ production profiles in anaerobic bioreactor batch cultures of $S$. cerevisiae strains with different genetic modifications in glycerol and acetate metabolism. Cultures were grown on synthetic medium containing $180 \mathrm{~g} \mathrm{~L}^{-1}$ glucose and $3 \mathrm{~g} \mathrm{~L}^{-1}$ acetic acid ( $\mathrm{pH}$ 5). A, IMZ160 (gpd1::IoxP gpd2::hphMX4 mhpF-overexpressing); B, IMX888

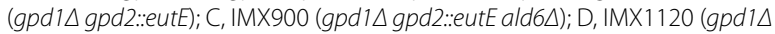
gpd2::eutE ald6 6 sga 1::ALD6); E, IMX1142 (gpd1::gpsA gpd2::eutE ald6 sga 1::ALD6). Data collected from online bioreactor offgas measurements. Representative cultures of independent duplicate experiments are shown.

Additional file 7. Starting and end concentrations of acetate and glycerol in anaerobic bioreactor batch cultures of S. cerevisiae strains IMX888 (gpd1 $\triangle$ gpd2::eutE) and IMX900 (gpd1 $\triangle$ gpd2::eutE ald6 4 ). Cultures were grown on synthetic medium containing $180 \mathrm{~g} \mathrm{~L}^{-1}$ glucose and $3 \mathrm{~g} \mathrm{~L}^{-1}$ acetate ( $\mathrm{pH}$ 5). Values represent averages \pm mean deviations of measurements on independent duplicate cultures.

Additional file 8. Potential cytosolic transhydrogenase cycle, exchanging $\mathrm{NADH}$ with NADPH, catalysed by EutE, Acs $1 / 2$ and Ald6. Formed NADPH can be used for DHAP reduction to glycerol by GpsA.

\section{Abbreviations}

G3PDH: glycerol-3-phosphate dehydrogenase; KPB: potassium-phosphate buffer; OD: optical density; A-ALD: acetylating-acetaldehyde dehydrogenase.

\section{Authors' contributions}

IP, AJAvM and JTP designed experiments and wrote the manuscript. IP selected the heterologous gene, constructed yeast strains and performed enzyme-activity 
assays and fermentation experiments. MvD constructed yeast strains, designed and performed fermentation experiments. All authors read and approved the final manuscript.

\section{Author details}

${ }^{1}$ Industrial Microbiology Section, Department of Biotechnology, Delft University of Technology, Van der Maasweg 9, 2629 HZ Delft, The Netherlands. 2 Present Address: Division of Industrial Biotechnology, Department of Biology and Biological Engineering, Chalmers University of Technology, Kemivägen 10, 41296 Gothenburg, Sweden. ${ }^{3}$ Present Address: Division of Industrial Biotechnology, School of Biotechnology, KTH Royal Institute of Technology, AlbaNova University Centre, 10691 Stockholm, Sweden.

\section{Acknowledgements}

We thank Jean-Marc Daran and Pilar de la Torre for advice on molecular biology, Matthijs Niemeijer and Erik de Hulster for advice on fermentations and Marijke Luttik for advice on enzyme-activity assays. We thank Donna Duiker for construction of strain IMX776 and our DSM colleagues for stimulating discussions.

\section{Competing interests}

IP, AJAvM and JTP are co-inventors in a patent application related to the present work.

\section{Availability of supporting data}

Codon-optimized sequences generated in this project were deposited in Genbank (http://www.ncbi.nlm.nih.gov/) (Accession Number: KY554758)

\section{Funding}

The Ph.D. project of IP is funded by DSM Bio-based Products \& Services B.V. (Delft, The Netherlands).

\section{Publisher's Note}

Springer Nature remains neutral with regard to jurisdictional claims in published maps and institutional affiliations.

Received: 21 February 2017 Accepted: 14 April 2017

Published online: 26 April 2017

\section{References}

1. Hermann BG, BlokK, Patel MK. Producing bio-based bulk chemicals using industrial biotechnology saves energy and combats climate change. Environ Sci Technol. 2007:41:7915-21.

2. Renewable Fuels Association. http://www.ethanolrfa.org. Accessed Nov 2016

3. Gombert AK, van Maris AJA. Improving conversion yield of fermentable sugars into fuel ethanol in 1st generation yeast-based production processes. Curr Opin Biotechnol. 2015;33:81-6.

4. Nielsen J, Larsson C, van Maris AJA, Pronk JT. Metabolic engineering of yeast for production of fuels and chemicals. Curr Opin Biotechnol. 2013:24:398-404.

5. Della-Bianca BE, Basso TO, Stambuk BU, Basso LC, Gombert AK. What do we know about the yeast strains from the Brazilian fuel ethanol industry? Appl Microbiol Biotechnol. 2013;97:979-91.

6. Hahn-Hägerdal B, Karhumaa K, Fonseca C, Spencer-Martins I, GorwaGrauslund MF. Towards industrial pentose-fermenting yeast strains. Appl Microbiol Biotechnol. 2007;74:937-53.

7. Moysés ND, Reis CV, Almeida RJ, Moraes ML, Torres AF. Xylose fermentation by Saccharomyces cerevisiae: challenges and prospects. Int J Mol Sci. 2016;17:207.

8. van Maris AJA, Abbott DA, Bellissimi E, van den Brink J, Kuyper M, Luttik MAH, Wisselink HW, Scheffers WA, van Dijken JP, Pronk JT. Alcoholic fermentation of carbon sources in biomass hydrolysates by Saccharomyces cerevisiae: current status. Antonie Van Leeuwenhoek. 2006;90:391-418.

9. Maiorella BL, Blanch HW, Wilke CR. Economic evaluation of alternative ethanol fermentation processes. Biotechnol Bioeng. 1984;26:1003-25.
10. Klinke HB, Thomsen AB, Ahring BK. Inhibition of ethanol-producing yeast and bacteria by degradation products produced during pre-treatment of biomass. Appl Microbiol Biotechnol. 2004;66:10-26.

11. Palmqvist $E$, Hahn-Hägerdal B. Fermentation of lignocellulosic hydrolysates. II: inhibitors and mechanisms of inhibition. Bioresour Technol. 2000;74:25-33.

12. Parawira W, Tekere M. Biotechnological strategies to overcome inhibitors in lignocellulose hydrolysates for ethanol production: review. Crit Rev Biotechnol. 2011;31:20-31.

13. Nissen TL, Kielland-Brandt MC, Nielsen J, Villadsen J. Optimization of ethanol production in Saccharomyces cerevisiae by metabolic engineering of the ammonium assimilation. Metab Eng. 2000;2:69-77.

14. van Dijken JP, Scheffers WA. Redox balances in the metabolism of sugars by yeasts. FEMS Microbiol Lett. 1986:32:199-224.

15. Verduyn C, Postma E, Scheffers WA, van Dijken JP. Physiology of Saccharomyces cerevisiae in anaerobic glucose-limited chemostat cultures. J Gen Microbiol. 1990:136:395-403.

16. Hohmann S. Osmotic stress signaling and osmoadaptation in yeasts. Microbiol Mol Biol Rev. 2002;66:300-72.

17. Nevoigt E, Stahl U. Osmoregulation and glycerol metabolism in the yeast Saccharomyces cerevisiae. FEMS Microbiol Rev. 1997;21:231-41.

18. Bakker BM, Overkamp KM, van Maris AJA, Kötter P, Luttik MAH, van Dijken JP. Pronk JT. Stoichiometry and compartmentation of NADH metabolism in Saccharomyces cerevisiae. FEMS Microbiol Rev. 2001;25:15-37.

19. Navarrete C, Nielsen J, Siewers V. Enhanced ethanol production and reduced glycerol formation in $f p s i \Delta$ mutants of Saccharomyces cerevisiae engineered for improved redox balancing. AMB Express. 2014;4:86.

20. Albertyn J, Hohmann S, Thevelein JM, Prior BA. GPD1, which encodes glycerol-3-phosphate dehydrogenase, is essential for growth under osmotic stress in Saccharomyces cerevisiae, and its expression is regulated by the high-osmolarity glycerol response pathway. Mol Cell Biol. 1994;14:4135-44.

21. Ansell R, Granath K, Hohmann S, Thevelein JM, Adler L. The two isoenzymes for yeast $\mathrm{NAD}^{+}$-dependent glycerol 3-phosphate dehydrogenase encoded by GPD1 and GPD2 have distinct roles in osmoadaptation and redox regulation. EMBO J. 1997;16:2179-87.

22. Larsson $K$, Ansell R, Eriksson P, Adler L. A gene encoding sn-glycerol 3-phosphate dehydrogenase $\left(\mathrm{NAD}^{+}\right)$complements an osmosensitive mutant of Saccharomyces cerevisiae. Mol Microbiol. 1993;10:1101-11.

23. Björkqvist $\mathrm{S}$, Ansell R, Adler L, Lidén G. Physiological response to anaerobicity of glycerol-3-phosphate dehydrogenase mutants of Saccharomyces cerevisiae. Appl Environ Microbiol. 1997:63:128-32.

24. Guadalupe-Medina V, Wisselink HW, Luttik MA, de Hulster E, Daran J-M, Pronk JT, van Maris AJA. Carbon dioxide fixation by Calvin-Cycle enzymes improves ethanol yield in yeast. Biotechnol Biofuels. 2013;6:125.

25. Guadalupe-Medina V, Almering MJH, van Maris AJA, Pronk JT. Elimination of glycerol production in anaerobic cultures of a Saccharomyces cerevisiae strain engineered to use acetic acid as an electron acceptor. Appl Environ Microbiol. 2010;76:190-5.

26. Wei N, Quarterman J, Kim SR, Cate JHD, Jin YS. Enhanced biofuel production through coupled acetic acid and xylose consumption by engineered yeast. Nat Commun. 2013;4:2580.

27. Xia PF, Zhang GC, Walker B, Seo SO, Kwak S, Liu JJ, Kim H, Ort DR, Wang SG, Jin YS. Recycling carbon dioxide during xylose fermentation by engineered Saccharomyces cerevisiae. ACS Synth Biol. 2016;6:276-83.

28. Zhang GC, Kong II, Wei N, Peng D, Turner TL, Sung BH, Sohn JH, Jin YS. Optimization of an acetate reduction pathway for producing cellulosic ethanol by engineered yeast. Biotechnol Bioeng. 2016;113:2587-96.

29. Papapetridis I, van Dijk M, Dobbe AP, Metz B, Pronk JT, van Maris AJA. Improving ethanol yield in acetate-reducing Saccharomyces cerevisiae by cofactor engineering of 6-phosphogluconate dehydrogenase and deletion of ALD6. Microb Cell Fact. 2016;15:67.

30. Henningsen BM, Hon S, Covalla SF, Sonu C, Argyros DA, Barrett TF, Wiswal E, Froehlich AC, Zelle RM. Increasing anaerobic acetate consumption and ethanol yields in Saccharomyces cerevisiae with NADPH-specific alcohol dehydrogenase. Appl Environ Microbiol. 2015;81:8108-17.

31. Koppram R, Tomás-Pejó E, Xiros C, Olsson L. Lignocellulosic ethanol production at high-gravity: challenges and perspectives. Trends Biotechnol. 2014;32:46-53

32. Puligundla P, Smogrovicova D, Obulam VSR, Ko S. Very high gravity (VHG) ethanolic brewing and fermentation: a research update. J Ind Microbiol Biotechnol. 2011:38:1133-44. 
33. Bayrock DP, Michael Ingledew W. Application of multistage continuous fermentation for production of fuel alcohol by very-high-gravity fermentation technology. J Chem Technol Biotechnol. 2001;27:87-93.

34. Ding WT, Zhang GC, Liu JJ. $3^{\prime}$ truncation of the GPD1 promoter in Saccharomyces cerevisiae for improved ethanol yield and productivity. Appl Environ Microbiol. 2013;79:3273-81.

35. Hubmann G, Guillouet S, Nevoigt E. Gpd1 and Gpd2 fine-tuning for sustainable reduction of glycerol formation in Saccharomyces cerevisiae. Appl Environ Microbiol. 2011;77:5857-67.

36. Thomas KC, Dhas A, Rossnagel BG, Ingledew WM. Production of fuel alcohol from hull-less barley by very high gravity technology. Cereal Chem. 1995;72:360-4.

37. Shen B, Hohmann S, Jensen RG, Bohnert HJ. Roles of sugar alcohols in osmotic stress adaptation. Replacement of glycerol by mannitol and sorbitol in yeast. Plant Physiol. 1999;121:45-52.

38. D'Amore T, Crumplen R, Stewart GG. The involvement of trehalose in yeast stress tolerance. J Ind Microbiol. 1991;7:191-5.

39. Mahmud SA, Nagahisa K, Hirasawa T, Yoshikawa K, Ashitani K, Shimizu H. Effect of trehalose accumulation on response to saline stress in Saccharomyces cerevisiae. Yeast. 2009;26:17-30.

40. Sharma SC. A possible role of trehalose in osmotolerance and ethanol tolerance in Saccharomyces cerevisiae. FEMS Microbiol Lett. 1997;152:11.

41. Sasano Y, Haitani Y, Ohtsu I, Shima J, Takagi H. Proline accumulation in baker's yeast enhances high-sucrose stress tolerance and fermentation ability in sweet dough. Int J Food Microbiol. 2012;152:40-3.

42. Takagi $\mathrm{H}$. Proline as a stress protectant in yeast: physiological functions, metabolic regulations, and biotechnological applications. Appl Microbiol Biotechnol. 2008;81:211.

43. Guadalupe-Medina V, Metz B, Oud B, van Der Graaf CM, Mans R, Pronk $J$ T, van Maris AJA. Evolutionary engineering of a glycerol-3-phosphate dehydrogenase-negative, acetate-reducing Saccharomyces cerevisiae strain enables anaerobic growth at high glucose concentrations. Microb Biotechnol. 2014:7:44-53.

44. Pagliardini J, Hubmann G, Alfenore S, Nevoigt E, Bideaux C, Guillouet SE. The metabolic costs of improving ethanol yield by reducing glycerol formation capacity under anaerobic conditions in Saccharomyces cerevisiae. Microb Cell Fact. 2013;12:29.

45. Sakasegawa SI, Hagemeier CH, Thauer RK, Essen LO, Shima S. Structural and functional analysis of the gpsA gene product of Archaeoglobus fulgidus: a glycerol-3-phosphate dehydrogenase with an unusual $\mathrm{NADP}\left({ }^{+}\right)$ preference. Protein Sci. 2004;13:3161-71.

46. Entian KD, Kötter P. 25 yeast genetic strain and plasmid collections. Methods Microbiol. 2007;36:629-66.

47. Verduyn C, Postma E, Scheffers WA, van Dijken JP. Effect of benzoic acid on metabolic fluxes in yeasts: a continuous-culture study on the regulation of respiration and alcoholic fermentation. Yeast. 1992;8:501-17.

48. Mans R, van Rossum HM, Wijsman M, Backx A, Kuijpers NG, van den Broek M, Daran-Lapujade P, Pronk JT, van Maris AJ, Daran J-M. CRISPR/Cas9: a molecular Swiss army knife for simultaneous introduction of multiple genetic modifications in Saccharomyces cerevisiae. FEMS Yeast Res. 2015;15:fov004

49. Wiedemann B, Boles E. Codon-optimized bacterial genes improve L-arabinose fermentation in recombinant Saccharomyces cerevisiae. Appl Environ Microbiol. 2008;74:2043-50.

50. Daniel Gietz R, Woods RA. Transformation of yeast by lithium acetate/ single-stranded carrier DNA/polyethylene glycol method. Methods Enzymol. 2002;350:87-96.

51. Solis-Escalante D, Kuijpers NGA, Nadine B, Bolat I, Bosman L, Pronk JT, Daran J-M, Pascale D-L. amdSYM, a new dominant recyclable marker cassette for Saccharomyces cerevisiae. FEMS Yeast Res. 2013;13:126.

52. Postma E, Verduyn C, Scheffers WA, van Dijken JP. Enzymic analysis of the crabtree effect in glucose-limited chemostat cultures of Saccharomyces cerevisiae. Appl Environ Microbiol. 1989;55:468-77.

53. Bryan AK, Goranov A, Amon A, Manalis SR. Measurement of mass, density, and volume during the cell cycle of yeast. PNAS. 2010;107:999-1004.

54. Albertyn J, Hohmann S, Prior BA. Characterization of the osmotic-stress response in Saccharomyces cerevisiae: osmotic stress and glucose repression regulate glycerol-3-phosphate dehydrogenase independently. Curr Gen. 1994;25:12-8.
55. Flikweert MT, de Swaaf M, van Dijken JP, Pronk JT. Growth requirements of pyruvate-decarboxylase-negative Saccharomyces cerevisiae. FEMS Microbiol Lett. 1999;174:73-9.

56. Saint-Prix F, Bönquist L, Dequin S. Functional analysis of the ALD gene family of Saccharomyces cerevisiae during anaerobic growth on glucose: the $\mathrm{NADP}^{+}$-dependent Ald6p and Ald5p isoforms play a major role in acetate formation. Microbiology. 2004;150:2209-20.

57. Blomberg A, Adler L. Roles of glycerol and glycerol-3-phosphate dehydrogenase $\left(\mathrm{NAD}^{+}\right)$in acquired osmotolerance of Saccharomyces cerevisiae. J Bacteriol. 1989;171:1087-92.

58. Zhang L, Tang Y, Guo ZP, Ding ZY, Shi GY. Improving the ethanol yield by reducing glycerol formation using cofactor regulation in Saccharomyces cerevisiae. Biotechnol Lett. 2011;33:1375-80.

59. Hasunuma T, Kondo A. Consolidated bioprocessing and simultaneous saccharification and fermentation of lignocellulose to ethanol with thermotolerant yeast strains. Process Biochem. 2012;47:1287-94.

60. Schuster BG, Chinn MS. Consolidated bioprocessing of lignocellulosic feedstocks for ethanol fuel production. Bioenergy Res. 2013;6:416-35.

61. Hirayarna T, Maeda T, Saito H, Shinozaki K. Cloning and characterization of seven cDNAs for hyperosmolarity-responsive (HOR) genes of Saccharomyces cerevisiae. Mol Gen Genet. 1995;249:127-38.

62. Varela JCS, van Beekvelt C, Planta RJ, Mager WH. Osmostress-induced changes in yeast gene expression. Mol Microbiol. 1992;6:2183-90.

63. Remize F, Cambon B, Barnavon L, Dequin S. Glycerol formation during wine fermentation is mainly linked to Gpd1p and is only partially controlled by the HOG pathway. Yeast. 2003:20:1243-53.

64. Edgley $M$, Brown AD. Yeast water relations: physiological changes induced by solute stress in Saccharomyces cerevisiae and Saccharomyces rouxii. Microbiology. 1983;129:3453-63.

65. Celton M, Goelzer A, Camarasa C, Fromion V, Dequin S. A constraint-based model analysis of the metabolic consequences of increased NADPH oxidation in Saccharomyces cerevisiae. Metab Eng. 2012;14:366-79.

66. Celton M, Sanchez I, Goelzer A, Fromion V, Camarasa C, Dequin S. A comparative transcriptomic, fluxomic and metabolomic analysis of the response of Saccharomyces cerevisiae to increases in NADPH oxidation. BMC Genom. 2012;13:317.

67. Grabowska D, Chelstowska A. The ALD6 gene product is indispensable for providing NADPH in yeast cells lacking glucose-6-phosphate dehydrogenase activity. J Biol Chem. 2003;278:13984-8.

68. González-Ramos D, de Vries ARG, Grijseels SS, van Berkum MC, Swinnen $S$, van den Broek M, Nevoigt E, Daran J-M, Pronk JT, van Maris AJA. A new laboratory evolution approach to select for constitutive acetic acid tolerance in Saccharomyces cerevisiae and identification of causal mutations. Biotechnol Biofuels. 2016:9:173.

69. Swinnen S, Fernández-Niño M, González-Ramos D, van Maris AJA, Nevoigt $E$. The fraction of cells that resume growth after acetic acid addition is a strain-dependent parameter of acetic acid tolerance in Saccharomyces cerevisiae. FEMS Yeast Res. 2014;14:642.

70. Saito H, Posas F. Response to hyperosmotic stress. Genetics. 2012;192:289-318.

71. Blomberg A. The osmotic hypersensitivity of the yeast Saccharomyces cerevisiae is strain and growth media dependent: quantitative aspects of the phenomenon. Yeast. 1997;13:529-39.

72. Singh KK, Norton RS. Metabolic changes induced during adaptation of Saccharomyces cerevisiae to a water stress. Arch Microbiol. 1991;156:38-42.

73. García MJ, Ríos G, Ali R, Bellés JM, Serrano R. Comparative physiology of salt tolerance in Candida tropicalis and Saccharomyces cerevisiae. Microbiology. 1997;143:1125-31.

74. Athenstaedt K, Daum G. 1-Acyldihydroxyacetone-phosphate reductase (Ayr1p) of the yeast Saccharomyces cerevisiae encoded by the open reading frame YIL124W is a major component of lipid particles. J Biol Chem. 2000;275:235-40

75. Mumberg D, Müller R, Funk M. Yeast vectors for the controlled expression of heterologous proteins in different genetic backgrounds. Gene. 1995;156:119-22

76. van Rossum HM, Kozak BU, Niemeijer MS, Duine HJ, Luttik MAH, Boer VM, Kõtter P, Daran J-M, van Maris AJA, Pronk JT. Alternative reactions at the interface of glycolysis and citric acid cycle in Saccharomyces cerevisiae. FEMS Yeast Res. 2016:16:017. 